Revista da ANPOLL, n" 4, p. 273-298, jan./jun. 1998

\title{
MYTHES FONDATEURS: L'ORIGINE DE L'INEGALITE (AFRIQUE NOIRE, AMERIQUE DU NORD)
}

\author{
Veronika Görök-Karady*
}

\begin{abstract}
RESSUME: Toule formation sociale élabore un certain discours plus ou moins consensuel sur l'expérience des inégalités afin d'en montrer les causes, de les justifier ou de les contester: Les discours sur l'inégalilé dans les sociélés sans tradition écrite s'objective dans la tradition orale. Ce texte présente d'abord l'histoire des enfants d'Eve - récit répandu en Europe, connu aussi au Brésil el en Afrique Noire. Ensuile, sont analy sés quelques exemples tirés des civilisalions d'Afrique Noire el des Noires d'Amérique.
\end{abstract}

MOTS-CLES: Tradition Orale; Inégalité; Mythes Fondateurs; Afrique Noire; Amérique du Nord.

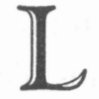
'inégalité entre les hommes est un des faits fondamentaux de la vie en société. On doit l'affronter dans la pratique quotidienne mais il faut la maitriser également sur le plan théorique pour la rendre tolérable en raison. C'est ainsi que dans toute formation sociale s'élabore un certain discours plus ou moins consensuel sur l'expérience des inégalités afin d'en montrer les causes, de les justifier ou de les contester. Dans les sociétés sans histoire écrite, très naturellement, ce discours s'objective dans la tradition orale. Celle-ci rend compte des inégalités comme d'une donnéc immanente à la condition sociale des hommes et en rejette les origines au début des temps. II s'agit le plus souvent de récits génétiques qui relient l'apparition des différences sociales à quelque incident primitif dans lequel furent impliqués les ancêtres. L'effet de cet incident demeurerait inamovible et déterminerait le destin collectif des hommes

* Pesquisadora do CNRS-Paris. Laboratório de Linguagem, Línguas e Culturas da África Negra. 
GÖRÖK-KARADY, Veronika. Mythes fondateurs: l'origine de l'inegalite (afrique noire, amerique du nord).

appartenant aux groupes ethniques, strates et races qu'on distingue notamment aujourd'hui. La prégnance de ce thème est telle qu'on en retrouve des élaborations dans des sociétés très diverses et jusque dans la tradition orale de sociétés industrielles. Pour montrer la généralité du thème nous commencerons par évoquer un récit de ce type, répandu en Europe, avant de passer en revue quelques exemples tirés des civilisations de l'Afrique Noire et des Noirs d'Amérique.

Un bon point de départ est offert par l'histoire des enfants d'Eve qui figure dans le catalogue d'Aarne-Thompson (AT 758). Ce conte religieux a pour objet la naissance des différences entre classes sociales ou, plus simplement, entre riches et pauvres. D'abord récit populaire, l'histoire a été très tôt reprise par des lettrés et reformulée plus d'une fois dans des genres littéraires savants, depuis la période de la Réforme. De fait il est facile de déceler dans ces exploitations du thème l'idéologie de la prédestination, chère à certaines mouvances de la Réforme, selon laquelle chacun doit se contenter de la place que Dieu lui a assignée sur l'échelle sociale: pour la bonne marche de l'ensemble toutes les fonctions doivent être remplies. Les privilégiés de cet arrangement portent toutefois une responsabilité supérieure, ce qui rétablit un certain équilibre dans l'économic morale de ces représentations collectives. En dehors des versions littéraires du thème on en connaît de nombreuses élaborations orales, ainsi en Allemagne, en Hongrie, en Catalogne, en Italie, à Malte, etc... Le récit est également connu au Brésil et en Afrique Noire, preuve qu'il a rencontré des résonances sous des climats sociologiques fort variés.

Toutes les versions européennes suivent essentiellement le même schéma, avancent des arguments semblables et véhiculent un message similaire. Voici comment le conte-type est présenté dans le catalogue d'Aarne-Thompson:

Eve a une si nombreuse progéniture qu'elle en a honte. Lorsque Dieu vient lui rendre visite, elle en cache un certain nombre. Les enfants non présentés 
Revista da ANPOLL, n" 4, p. 273-298, jan./jun. 1998

n'obtiendront pas la bénédiction divine accordée aux autres. Ceci explique les différences entre classes sociales, groupes et peuples.

Sans entrer ici dans l'analyse détaillée du récit - qui a été tentée ailleurs (Görök-Karady, 1982) - il convient d'en noter les principaux éléments: son aspect misogyne (Eve, mère des humains, est fautive), son rattachement au thème biblique du péché originel, enfin le fait capital variablement mis en valeur dans les différentes versions -: Dieu, aussi omniscient et tout-puissant soit-il, se refuse à toute intervention pour annuler ou compenser le préjudice que la faute maternelle attire sur les enfants "non présentés". Ce refus subsiste même dans les versions où Eve essaie de se rettraper et sollicite sur le tard la bénediction divine pour les enfants "cachés". Ce refus semble inćbranlable et condamme la progéniture malheureuse au destin des mal-lotis: "...maintenant le monde est divisé et ce qui est fait est fait. Les douze autres enfants n'auront pas le choix et devront gagner leur vie sur les terres des douze premiers enfants... C'est ainsi qu'il y a des riches et des pauvres sur terre".

Tout se passe donc au témoignage du conte, comme si la division inégalitaire du monde social ressortissait, tout au moins indirectement, à la volonté de Dieu qui en avalise la responsabilité. Pareille conception s'inscrit dans le problème de l'intégration du Mal dans le système religieux judéo-chrétien: Dieu laisse les hommes libres de faire le mal tout en les exhortant à faire le bien. Le message idéologique du récit est de la sorte double: d'une part consolateur, puisque les descendants ne partagent point la faute originelle, d'autre part portant à la résignation, puisqu'il convient d'accepter la volonté de Dieu.

Cette rapide évocation d'un récit d'origine traditionnel qui peut être considéré comme le modèle du genre en Europe, permet de passer à l'étude des récits idéologiques apparentés en Afrique Noire et en Amérique dans l'espoir d'en dégager des marques structurelles communes relevant de la logique idéologique particulière qui anime ce type de littérature orale. 
GÖRÖK-KARADY, Veronika. Mythes fondateurs: lion sine de l'inegalite (afrique noire, amerique du nord).

\section{RÉCITS D'AFRIQUE NOIRE}

Les récits consacrés à l'origine des relations inégalitaires entre Blancs et Noirs, se retrouvent dans un grand nombre de sociétés au Sud du Sahara, si bien qu'on peut sans grand risque d'erreur conclure à leur généralité dans cette zone de civilisation. Le message que portent ces récits renvoie à l'instar de l'idée centrale de l'histoire des enfants d'Eve en Europe - à la condition aliénée des colonisés. De même, ils se réfèrent également à des incidents survenus dans les temps immémoriaux et proposent des explications tout aussi justificatrices et consolatrices quant à l'état des choses établies. Leur caractère idéologique est donc manifeste. Pour les présenter brièvement, voici un classement simple qui permet de ranger ces textes oraux en trois groupes selon la nature du message qu'ils véhiculent:

- récits qui interprètent la différenciation raciale en termes d'accident ou de hasard;

- textes qui imputent celle-ci à l'intervention (non provoquéc) d'un tiers, à l'action arbitraire de Dieu ou du parent ancestral;

- textes qui mettent en jeu la responsabilité des hommes, en particulier des ancêtres africains.

Ce classement élémentaire offre un aperçu significatif de corpus: de fait, la majorité des textes se range dans le troisième groupe et glose sur la "faute primitive" engageant une responsabilité ancestrale dans le destin des descendants. I _e classement indique aussi les grandes structures narratives dans lesquelles sont logés les messages idéologiques relatifs aux causes de l'inégalité sociale. I_es textes des deux premiers groupes, reflétant une vision fataliste de l'histoire sont aussi les plus courts et de structure relativement simple (sans rebondissements). I.es récits avec responsabilité sont plus complexes.

Dégagéc à partir d'une centaine de textes, voici une vue d'ensemble de l'agencement narratif des récits de genèse africains: 


\section{SITUATION D'OUVERTURE}

a) L'humanité n'existe pas, une divinité décide souverainement de sa création.

b) L'humanité primordiale existe, elle est indifférenciée.

- Les premiers humains (dont le nombre peut varier de deux à un nombre indéfini) sont soit tous noirs, soit tous blancs, ou encore d'une coulcur non spécifiée.

- I Les premiers humains sont différenciés quant à la couleur de leur peau, mais les dons intellectuels et les statuts socioéconomiques restent à distribuer:

c) L'humanité primordiale existe, elle est différenciée.

- L’un des ancêtres (le Noir ou le Blanc) est le préféré de Dieu ou de ses parents.

- L'un des ancêtres (le Noir ou le Blanc) détient une position supéricure, par exemple par droit d'aînesse.

- Les deux ancêtres sont d'essence différente (le Blanc est d'essence divine).

\section{DÉROULEMENT}

I - La responsabilité humaine n'est pas engagée dans l'histoire.

a) I.e hasard décide du destin des races, il favorise les Blancs. (Exemple: un des frères trouve de l'eau, il se baigne, devient blanc; pour l'autre, il ne reste que peu d'eau, il sera noir. I_e Blanc sera heureux, le Noir malheureux). 
CÖRÖK-KARADY, Veronika. Mythes fondateurs: l'origine de l'inegalite (afrique noirr, amerique du nord).

b) Dieu crée les ancêtres, décide de leur sort et de celui de leur descendance. Sa décision est arbitraire, il n'agit pas en fonction des mérites. Cette modalité de différenciation est narrée de façon simple: l'être supérieur étant l'agent, les humains n'ont qu'à subir son action.

\section{II - La responsabilité humaine est engagée dans l'histoire}

c) Différenciation par l'éprèuve:

- Epreuve du bain. Un être divin ou un autre agent ordonne aux héros de se baigner dans un lac froid, de traverser un fleuve aux eaux sales. Il les récompense en fonction de leurs perfomances. L'issue de l'épreuve marquera toute la descendance des participants, leur assignant des rôles et des conditions sociales déterminées par la réussite ou l'échec de l'ancêtre. l'épreuve peut avoir lieu sur l'initiative d'un des ancêtres, sans que Dicu (ou un parent) intervienne pour la provoquer.

- Epreuve de choix. Un être divin ou un parent ordonne aux héros d'effectuer un choix (habituellement il faut choisir entre deux tas d'objets). Dans tous les récits répertoriés ce sont les héros noirs qui choisissent en premier. Dans chaque cas ceux-ci font un mauvais choix qui entraîne leur dégradation: ils se fient aux apparences, cherchent la satisfaction immédiate, suivent le principe du moindre effort. (Ils dédaignent par exemple le livre, objet qui assure à celui qui le possède la maîtrise des techniques complexes et la supériorité économique). Ce choix des Noirs est désapprouvé de façon plus on moins violente: si le mécontente- 
ment divin est grand, Dieu les châtic en les reléguant sous la tutelle des Blancs.

Notons encore à propos de ces mythes que les héros sont, dans un certain nombre de récits, prévenus à l'avance de l'enjeu de l'épreuve, fait qui accrôt leur responsabilité. Dans les autres cas, ils ignorent l'importance que prend pour eux l'épreuve.

d) Différenciation résultant d'un méfait ou d'une négligence, d'une faute de comportement. Les destins des deux humanités peuvent être diversifiés à la suite d'un acte malfaisant ou du fait d'un manquement à un devoir. Dans les contes à épreuve, comme on l'a vu, les représentants des deux races sont mis dans une situation identique: tous deux sont appelés à l'action et répondent à cet appel. Par contre, dans la majorité des récits à méfaits, scul un des deux héros agit.

- Le héros ancêtre commet une faute religicuse ou sociale, par exemple en désobéissant à Dicu, en rompant un interdit, en maltraitant un compagnon ou en essayant de commettre un meurtre. L'autre protagoniste, du scul fait qu'il s'abstient de commettre le même méfait ou qu'il adopte un comportement inverse dans la même situation, sera gratifié par Dicu ou un parent et bénéficiera de la suprématic.

- L'un des héros se montre négligent à l'égard des règles de la bienséance, en oubliant par exemple de remercier Dicu pour un bienfait. Là encore c'est son rival qui s'en trouve avantagé.

Signalons ici que la différenciation par épreuve et celle résultant d'un méfait ou d'une négligence peuvent figurer dans un seul et même récit. 
CÖ̈RÖK-KMRNI)Y, Veronika. Mythes fondateurs: l'orgine de l'inegalite (afrique noire, amerique du nordl).

\section{SÉQUENCE FINALE}

Dans la séquence finale le conteur exprime souvent son point de vue sous forme de commentaires et de conclusion. Dans les récits plus récents on trouve des réflexions plus élaborées. La plus grande partie des récits traduisent la résignation des Noirs. I a contestation de la domination blanche d'une façon non violente et sur le mode mythique est l'étape suivante. 'Tì̀s rares sont les textes où l'I lomme Blanc est haï et les valeur's curopéennes rejetées.

\section{I - Récits qui manifestent une idéologie de résignation}

- La supériorité des Blancs est annoncéc, elle est légitimée par l'exploit de leur ancêtre mythique.

- ILe héros noir ayant commis une faute grave exprime, dans la conclusion du conte, un sentiment de culpabilité prononcée. Le caractère irrémédiable de la condamnation est souligné. A cette catégoric appartiennent notamment les récits où les Noirs sont en position supéricure en début de récit (en tant qu'aînés par exemple) et, de par leur faute, perdent cet avantage.

- Dans les récits où l'inégalité est instaurée de façon arbitraire, l'état de subordination des Noirs est présenté comme définitif dans la conclusion.

\section{II - Récits qui manifestent une idéologie de contestation}

- La contestation de la supériorité blanche ne débouche pas sur l'hostilité. On reconnait la supériorité des Blancs, on se propose de devenir leur égal et on sollicite leur coopération. L.es "connaissances", symbolisées par l'écriture et le livre, sont hautement appréciées. 
- Quelques récits sculement portent une accusation violente contre les Blancs. Dans ces récits, le narrateur disculpe les Noirs et accuse les Blancs d'être des malfaiteurs rusés.

La cohérence interne du corpus africain - dont les récits aussi divers soient-ils quant aux thèmes mis à contribution, se ramènent finalement à peu d'options alternatives - pousse à l'examen d'autres représentations de l'inégalité sociale dans la tradition orale, telles qu'elles s'expriment ailleurs qu'en Afrique. Depuis peu nous disposons sur cette question d'un riche matériel complémentaire grâce au travail inédit d'une jeune anthropologue, Patricia Turner, qui a réuni vingt-sept récits étiologiques chez des Noirs des Etats-Unis.

\section{RÉCITS DES NOIRS AMÉRICAINS}

Avant de confronter ce matériel avec le corpus africain, quelques remarques préalables s'imposent.

II faut dire tout d'abord que, en dépit des fortes similitudes qui se manifestent entre ces deux corpus, il n'y a pas lieu de supposer que les récits américains soient d'origine africaine et "importés" d'Afrique. I.es contacts entre Blancs et Noirs en Afrique, avant que les futurs esclaves fussent arrachés à leur terre natale, devaient être en général par trop sporadiques pour pousser les Africains à l'élaboration d'un discours cohérent sur eux-mêmes en fiace des Blancs. II est bien plus probable que ce type de discours ait pris naissinnce dans la situation coloniale établie en Afrique et dans la condition faite aux esclaves au Nouveau Monde. De la sorte, si les deux discours présentent des parallélismes, il convient de les attribuer à l'expérience vécue similaire de l'oppression des Noirs par des Blancs.

Les ressemblances constatées s'expliquent très probablement par le fait que les textes reflètent des valeurs directement reprises aux Blancs dominateurs. Il s'agit donc, dans les deux cas, non sculement de la 
GÖRÖK-KARADY, Veronika. Mythes fondateurs: lon sine de l'inegalite (afrique noirr, amerique du nord).

rationalisation d'une expérience collective mais aussi de la réception de l'idéologie des dominants. On ne manque pas en effet de témoignages, en Afrique comme en Amérique, sur les cas de transmission et d'inculcation des idées "européennes" sur les qualités des "races", dont les principaux agents étaient aussi divers que puissants (missions, écoles, administration, autorités judiciaires et militaires). On sait qu'ici et là les prédicateurs, pendant longtemps exclusivement blancs, n'hésitèrent pas à manipuler les textes bibliques devant des auditoires noirs pour escamoter les passages portant sur la liberté et mettre l'accent sur les thèmes moralisateurs impliquant le devoir de soumission. On comprend ainsi la forte prégnance que gardent les thèmes qui renforcent l'intériorisation de l'idéologie des Blanes dans le discours traditionnel des Noirs des deux côtés de l'Atlantique.

S'il en est ainsi, il convient toutefois de ne pas oublier que pareille acceptation d'une idéologie aliénante n'avait rien d'absolu ni de définitif. Ces idées reçues ont été et sont susceptibles de modifications, voire de retournements dans le cadre d'un même discours traditionnel. Ainsi les mêmes schémas narratifs peuvent porter également des messages dirigés contre les dominateurs, une fois vidés de leurs intentions primitives.

Rappelons enfin que le destin social des Africains et des Noirs d'Amérique accuse une différence fondamentale quant à l'expérience de l'aliénation historique qui leur a été infligéc, dans la mesure où les premiers ont su conserver sous le règne colonial une large autonomic culturelle, voire le plus souvent les formes traditionnelles de leur organisation communautaire, tandis que les derniers ont souffert d'un déracinement complet dans l'esclavage, au point de se voir priver de leur langue, leur religion, leurs structures familiales, etc... En comparant les textes idéologiques des Noirs des deux côtés de l'Atlantique, on compare donc l'empreinte laissé dans la tradition orale de deux situations largement différentes dont il faudrait rechercher, et aussi, en bonne logique, retrouver la trace littérairc.

Certaines de ces différences affleurent dans l'agencement narratif des thèmes de base. 
Ainsi, alors que les récits génétiques africains s'attachent aux éléments de l'altérité des groupes en présence, notamment aux traits physiques et moraux des Blancs, les narrations afro-américaines s'intéressent bien davantage aux seuls Noirs. On comprend que, en Afrique Noire, les Européens venus d'un continent inconnu, dotés e caractères culturels et d'une civilisation technique aux ressorts insaisissables, enfin demeurant dans une distance considérable dans l'espace social par rapport aux populations colonisées, aient vite fait de constituer un véritable objet de curiosité. En Amérique au contraire, quelque radicale qu'ait été l'inégalité de condition entre Noirs et Blancs ils ne cessaient de vivre ensemble dans cette symbiose singulière des maitres et des esclaves d'abord, dans le champ de tensions des oppositions de classe ensuite, mais partageant toujours la même civilisation "chrétienne" et occidentale qui a été imposée aux Noirs. En Amérique les autochtones étaient les Blancs. I_es Noirs transplantés ont été contraints à une assimilation forcée qui les a, objectivement, intégrés dans la société américaine et rapprochés ainsi des Blancs. Ces derniers n'ont pas pu présenter la même figure d'étrangeté fabuleuse en Amérique qu'en Afrique, d'où l'impact relativement faible de la représentation des Blancs dans les récits des Noirs d'Amérique.

Une autre différence manifeste entre les deux corpus est constituée par la présence de récits de genèse à tonalié humoristique aux Etats-Unis, ce qui semble faire totalement défaut en Afrique. Les figures d'ironic sont toujours des formes distanciées des rapports aux choses, ici du rapport à soi et à l'identité collective. C'est aussi l'expression d'un déplacement des objets de litige, comportant un certain dépassement du litige lui-même, du moins son euphémisation que connaissent bien des groupes dominés, tels les Juifs européens de jadis (et de toujours). Tourner en ridicule leur destin historique, par ailleurs tragique, n'est-pas, pour les Noirs d'Amérique une manière de se libérer de la pesanteur de ce destin et d'affirmer une relation déjà distendue avec l'héritage collectif du passé? Ceci soulève le problème de la transformation du status de ces récits traditionnels. II semble 
GÖRÖK-KAR NDY, Veronika. Mythes fondaleurs: lion gine de l'inegalite (afrique noire, amerique dn nord).

évident qu'on ne raconte pas de la même manière les mêmes histoires et surtout qu'on ne leur attribue pas la même signification dans des situations historiques différentes devant des publics eux aussi profondément modifiés. Pour interpréter la nature des modifications, il faudrait disposer d'informations "contextuelles" précises sur les conditions de production des récits étiologiques, sur leur réception, sur la qualité des narrateurs et de leur auditoire. L'ironic qui perce dans certaines narrations au contenu idéologique bien grave vient peut-être du fait que ces récits "reflect what the Black American thinks the White American thinks about the Black American", d'après la formule qui conclut l'analyse c Patricia Türner fondée sur les informations contextuelles qui accompagnent les textes enregistrés depuis 1960.

\section{RÉSUMÉS DES TEXTES AFRO-AMÉRICAINS:}

I - Textes où la responsabilité humaine n'est pas engagée. (Dieu décide ou le hasard).

1. Dieu créa les premiers Noirs, Indiens, Chinois... de la terre. II lui restait encore deux hommes à faire, l'Anglais et le Français, mais comme il n'y avait plus de terre qui lui convint, il utilisa de la "matière vivante", un papillon et une fourmi. C'est pourquoi, faits de matière douée de vie, l'Anglais et le Français réussirent micux dans la vie que les autres.

Informateur: ancien esclave, région du Mississipi, cf. A. F. Chamberlain "Negro Creation Legend", Journal of American Folklore, 1980, 3, p. 302.

2. Autrefois tous les hommes étaient noirs. Un petit garçon tomba dans un baquet plein de lessive faite de cendres et devient tout blanc. Plus tard, ses enfants devinrent également blancs. Voilà l'origine des Blancs. 
Informateur: étudiant qui l'a entendu raconter par sa mère en 1935: celle-là a dû entendre vers 1900 dans l'état de Tennessec par une femme noire travaillant dans leur ferme.

3. Les Noirs ont la peau de couleur foncée car autrefois ils ont vécu dans un licu très chaud (bronzagc).

Informateur: étudiant blanc auquel la mère donna cette explication en 1950 en réponse à sa question.

4. Les Noirs ont les cheveux crépus car Dieu, chaque fois qu'il créait un enfant noir, était très fâché et dans sa colère il roussissait ses cheveux.

Informateur: étudiant blanc de Berkeley qui l'a entendu dire par son père qui lui aussi l'avait entendu raconter par son père dans la région de Mississipi vers 1909.

5. Dieu, en voyant sa créature, le Noir, dit: "Zut,j'en ai encore brûlé un!" Informateur: étudiant blanc qui a entendu cette anecdote à l'école primaire en 1955.

6. Dieu, en créant le second Noir, dit: "Zut, j’ai encore brûlé celuilà!"

Informateur: étudiante blanche; elle l'a entendu dire par sa socur en 1963.

7. Dieu décida de créer les hommes à partir de pain d'épice à cause de son goût agréable. Mais il sortit la première fournée d'hommes trop tôt, ils sont restés trop pâles, trop peu cuits. Ils sont devenus les Blancs. Les hommes de la seconde fournée n'étaient toujours pas assez cuits. Ce sont les Chinois. Lees hommes de la dernière fournée, Dieu les laissa cuire plus longtemps, ceux-là sont de couleur foncée comme il le souhaitait. II les appela les nègres parce qu'ils étaient les meilleurs. 
CÖ̈ÖK-KARNI)Y, Veronika. Myıthes fondateurs: lomigine de l'inegalite (afrique noirr, amerique du nord).

Informateur: étudiante afro-américaine; elle a entendu ce récit de sa mère vers 1953.

II - Textes où la responsabilité humaine est partiellement ou totalement engagée.

8. Autrefois, tous les hommes, etaient noirs. La nouvelle s'était répanduc qu'il existait un lac dont l'eau transformait ceux qui s'y baignaient en blancs et changeait les cheveux crépus en cheveux lisses. Effectivement les premiers noirs qui arrivèrent se transformèrent en blancs, ceux qui arrivèrent plus tard quand il y avait déjà moins d'eau se transformèrent en mulâtres et les derniers arrivés ne trouvèrent plus qu'un peu d'eau d'où la blancheur scule de la paume de leurs mains et de la plante de leurs pieds.

Informateur: ancien esclave qui a passé toute sa vie dans l'état de Virginie; date de l'enquête: années 1930.

9. Au début tout le monde était noir: Un jour, Dieu dit aux hommes d'aller se baigner dans une rivière pour qu'ils deviennent blancs et que leur cheveux devinssent lisses. La moitié des gens obéirent, les autres restèrent allongés et rirent. Quand ils curent vu les autres transformés, ils se levèrent mais il ne restait que très peu d'eau et seules la paume de leurs mains et la plante de leurs pieds devinrent blanches.

Informateur: femme de ménage afro-américaine; elle a appris ce récit de sa mère vers 1930; cette dernière a vécu dans l'etat de Caroline du Sud.

10. Une fois la rumeur se répandit que l'eau d'un lac transformait les gens de noirs en blanes. I es Noirs de la région s'empressèrent d'y aller dans l'espoir de pouvoir résoudre leurs problèmes grâce 
au bain. Comme ils étaient nombreux, l'eau ne suffit pas et ceux qui arrivèrent plus tard ne blanchirent que la paume de leurs mains et la plante de leurs pieds.

Informatcur: étudiant blanc qui a entendu l'histoire d'un Noir cn 1966.

11. Autrefois tous les hommes étaient de couleur marron. Dieu désira alors que les gens soient de couleur différente et leur ordonna de se baigner dans un fleuve. Ceux qui se sont baignés longtemps sont devenus les Blancs, ceux qui se sont baignés brièvement sont devenus les Orientaux et ceux qui y ont juste trempé les mains et les pieds sont devenus les Noirs.

Informateur: instituteur noir, l'a entendu dire à son père vers 1952.

11b. Certains Africains ont vécu dans la jungle en Afrique. Près de leur village, il y avait un lac dont on croyait qu'il possédait des vertus magiques. Un jour; les gens sont entrés dans le lac, ont pris de l'eau dans la main pour boire. C'est la raison pour laquelle les Noirs ont la paume de la main, la plante du pied et la langue blanches.

Informatrice: écolière noire originaire du sud des Etats-Unis; clle l'a raconté en 1962 à New-York.

11c. Tous les hommes étaient noirs à l'origine et ceux qui par la suite devinrent blancs étaient les plus sensés. L’Ange du Seigneur descendit du Ciel pour ordonner à tous les hommes d'aller se laver le soir de vendredi dans l'eau du Jourdain pour qu'ils fussent blanes et que leurs cheveux fussent lisses. Mais les Noirs stupides n'écoutèrent pas l'Ange qui répéta pourtant le message à plusieurs reprises. Ceux qui arrivèrent à temps devinrent blancs.

Recueilli dans les années 1930. 
CÖRÖK-KARADY, Veronika. Mythes fondateurs: lion :gine de l'inegalite (afrique noive, amerique du nord).

III - Textes où la responsabilité humaine est engagée.

12. Dieu décida de distribuer aux hommes leur chevelure. II appela le Chinois, le Mexicain, le Blanc qui interrompirent immédiatement leur jeu de hasard. I.es hommes noirs, qui étaient en train de gagner dans le jeu, ne s'arrêtèrent pas. Ils demandèrent à Dieu de rouler leur chevelure en boule. Ce que Dieu fit.

Informateur: étudiant afro-américain à Berkeley; il a entendu I'histoire la première fois à St-Louis, Missouri, en 1959.

13. Au début, les gens étaient laids: ils n'avaient pas de cheveux. Dieu décida alors de les rendre beaux et les invita a manger des melons pour qu'il puisse leur distribuer leur chevelure. Ils mangaient et s'amusaient ensemble et Dicu les appelait au fur et a mesure. Tous se présentaient immédiatement quand Dieu les appelait sauf les Noirs qui mangeaient leurs melons. Quand enfin ils se présentèrent, il ne leur resta plus que la chevelure crépue dont personne ne voulait et que tout le monde foulait aux pieds.

Informateur: étudiant afro-américain qui a entendu ce récit en 1929 pour la première fis, alors raconté par sa mère.

14. Au début, quand Dieu créa les gens, il demandait à chaque race quel type de cheveux elle voulait avoir. Ice Blanc, le Chinois et le Mexicain dirent ce qu'ils voulaient avoir. Le Noirs se leva difficilement et moitić somnolent dit à Dieu: "Roule-moi en boule et lance-moi ce que tu trouves sous la main". Et Dicu fit ainsi. Informateur: étudiante afro-américaine qui tient l'historie de son père né en 1921 dans l'état de Louisiane.

15. A l'origine, personne n'avait de cheveux et les gens étaient laids. L'ordre fut alors donné à tout le monde de se présenter à la 
distribution de cheveux. Les gens s'amusaient et mangeaient des melons mais les Indiens, les Chinois et les Blancs interrompirent à l'appel divin leur jeu et laissèrent les melons. Quand les Noirs arrivèrent, la seule chevelure qui restait gisait par terre, roulée en boule dans un grand désordre.

Informateur: travailleur noir de 57 ans à Berkeley. Il l'a appris de son père, natif de la région du delta du Mississipi en 1969.

16. Dieu distribua la chevelure aux hommes. Il travaillait dur et donna à l'Oriental et au Blanc ce qu'ils demandaient. Quand ce fut le tour du Noir, ce dernier se leva et dit: "Il fait noir, je sais que vous avez travaillé durement Dieu, prenez mes cheveux et lancez-les-moi ici".

Informateur: étudiante afro-americaine de 21 ans, a appris l'histoire de ses amis en Californie.

17. Au début, les gens n'avaient pas de cheveux et ils étaient tous très heureux. Puis ils décidèrent qu'ils voulaient quand même en avoir. Ils organisèrent une fête et s'amusaient et mangeaient du melon. Quand il fallait aller chercher leur chevelure, les Blancs, les Chinois et les Indiens qui n'aimaient pas tant le melon y allaient à temps tandis que les Noirs tardaient. Il ne leur resta que les cheveux écrasés par terre.

Informateur: étudiante afro-américaine; elle tient le récit de sa mère qui le lui raconta vers 1961.

18. Dieu ne créa pas les hommes en une seule fois: un jour, il distribua les yeux, un autre jour les dents et un autre la couleur. Le jour où il donna la couleur les Noirs étaient très en retard et il envoya les Anges pour les chercher. Comme les Noirs se pressaient ils se donnaient des coups; Dieu leur dit alors: "Get back, get 
CÖRÖK-KARNDY, Veronika. Mythes fondaleurs: liorigine de l'inegalite (ufrique noire, ammerique du nord).

back!" mais ils comprirent mal (ils entendirent "get black!") et c'est pourquoi ils devinrent noirs et le sont restés depuis.

Informateur: jeune femme noire, dans les années 1930, Eatonville, en Floride.

19. Dieu envoya l'ange Cabricl chercher les Noirs. II finit par les trouver endormis sous l'Arbre de la Vie. Quand ils reçurent le message divin, de peur de ne pas avoir leur part, ils se mirent à se bousculer les uns les autres. Dieu leur cria alors: "Cet back!" mais ils le comprirent mal, devinrent "black" et le sont restés depuis. Informateur: jeune Noir d'Eatonville, dans les annécs 1930 (même session que $\mathrm{n}^{0} 18$ ).

20. Dieu convoqua les hommes pour leur donner leur chevelure. Tout le monde arriva sauf le Noir qui dormait dans un coin et qui n'entendit pas l'appel. Quand Dicu l'aperçut, il l'appela mais le Noir n'avait pas envie de se lever et demanda à Dieu de lui lancer sa chevelure. Dieu alors se fâcha, roula les cheveux en boule et les lui lança. C'est pourquoi les Noirs ont les cheveux crépus. Informateur: étudiate afro-américaine qui a entendu ce récit la première fois en 1949 dans l'état de Caroline du Nord; elle le raconte en 1974.

21. Autrefois un homme demanda au Blanc, à l'Indien, au Mexicain ct au Noir quelle chevelure ils voulaient avoir: Pour recevoir ce qu'ils voulaient, il fallait faire cing miles a pieds dans la montagne. I.e Blanc, l'Indien et le Mexicain acceptèrent les conditions et ils reçurent la chevelure demandée, mais le Noir dit que c'était trop loin et qu'il fallait plutôt rouler la chevelure en boule et la lui lancer. Informatcur: jeune fille anglaise de 17 ans; elle l'a racontée en 1971 à Berkelcy. 
22. Dieu décida de créer les hommes. II fit d'abord les yeux, les dents, les jambes et les pieds. Pour la couleur; il les convoqua pour sept heures du matin. 'Toute le monde y était excepté les Noirs. Il attendit en vain et finit par envoyer les Anges les chercher: Après de longs efforts, ccux-là finirent par les trouver allongés sous un arbre au soleil. L_es Anges les réveillèrent et leur remirent le message divin. Les Noirs se levèrent, bousculèrent les Anges et les gens sur leur passage et même le trône de Dicu. Dicu leur cria: "Cet back!". Ils comprirent: "Get black!" et devinrent noirs. Informateur: dame de 84 ans. Elle le raconte à Pittsburg (Californic) en 1967 à sa petite-fille afro-américaine.

23. Au début des temps, les gens n'avaient pas de cheveux. Ils furent donc convoqués pour la distribution de chevelure. En attendant, ils organisèrent une partic de pastèque.

Quand leur tour arriva, les Chinois, les Japonais et des Blancs se mirent en avant mais les Noirs ne voulaient pas laisser la pastèque. Quand ils se présentèrent enfin, les cheveux lisses avaient déjà été distribués et la scule chevelure dont personne ne voulait gisait par terre, écraséc par les gens qui marchaient dessus. C'est ainsi que les Noirs ont les cheveux crépus. Informateur: pompier, résident d'une ville noire isoléc: Calvin. Date de la collecte: 1952. I Le collecteur R. Dorson souligne la tension existant à l'intéricur de la communauté noire entre les gens de teinte plus claire, les plus ambiticux, et ceux de teinte plus foncée qui l'étaient moins.

24. Texte reproduit dans le corps de l'article.

Informateur: anciennement esclave dans les états d'Alabama et de Caroline du Nord. 
GÖRÖK-KARADY, Veronika. Mythes fondateurs: lion gine de l'inegalite (afrique noire, amerique du nord).

25. Caïn tua son frère Abel et quand Dieu lui en demanda des comptes, la peur le fit se transformer en blanc: c'était en fait la marque de Dieu. Il fut aussi obligé d'aller s'établií dans une autre contrée.

Informateur: esclave prédicateur, occupant donc le poste hiérarchique le plus élevé chez les Noirs.

Formellement la distinction entre récits à responsabilité assignée aux ancêtres et les autres histoires étiologiques sur l'inégalité est autant opératoire en Amérique qu'en Afrique. Ici encore le dernier groupe comporte des textes plus brefs, moins élaborés et d'ailleurs moins nombreux.

On remarquera que, du point de vue thématique, la matière des récits américains "sans responsabilité humaine" est en tout point identique à celle de leurs analogues africains. Ici encore la différenciation raciale est soit le fruit d'accident, notamment d'accidents écologiques ou climatiques (Noirs plus "bronzés"), soit relève de la "mauvaise création" (Noirs fabriqués à partir d'animaux ou de la matière inanimée) ou d'une création franchement ratée. Tous ces éléments se retrouvent sous des formes à peine différentes en Afrique. Ces récits étiologiques s'inscrivent des deux côtés de l'Atlantique dans un jeu de variations sur un stock thématique très reduit.

Dans le second grand ensemble aussi, marqué par l'idée de la responsabilité ancestrale, les textes puisent largement dans les schémas connus des épreuves du bain ou du choix.

En Amérique aussi l'épreuve du bain permet de "blanchir" les hommes inégalement dans tous les sens du mot. L'interprétation d'une pigmentation claire est ici encore celle d'un heureux événement. Notons que le message de ces récits oscille entre le fatalisme (accident écologique) et la culpabilité primitive. Tout se passe parfois comme si les ancêtres étaient tenus à répondre de ce qui leur arrive (et à leurs descendants) lors même qu'ils ne sont pas conscients, c'est-à-dire informés de l'enjeu de leur acte. Leur "faute" est en tout état de cause hors de proportion avec le 
sort qu'elle attire sur la descendance, ce qui fait dès lors apparaître celuici comme une condamnation, plutôt que comme le résultat d'une épreuve. On se souvient d'ailleurs que, en classant nos textes africains de ce type, nous leur avons attribué une inspiration initiatique. En effet le passage par un cours d'eau ou l'immersion dans un lac s'apparentent dans l'imaginaire africain aux actes de mort et de renaissance symboliques. Dans le cas présent la transformation provoquée par l'épreuve induit la disparition de l'humanité indifférenciée et l'émergence des groupes raciaux et sociaux inćgaux. II s'agit ici aussi d'un acte fondateur de l'histoire qui confère aux hommes leur nature sociale. Si le support culturel de cette représentation de la genèse, en tant qu'acte iniatique, n'existe guère en Amérique, les récits bâtis sur ce schéma n'en gardent pas moins l'essenticl de leur sens primitif: une mutation par le passage dans l'eau.

Les récits avec épreuve de choix font en Amérique figure originale parce que l'option proposéc aux premiers humains concerne ici exclusivement la qualité de la chevelure (cheveux crépus contre lisses), thème qui n'a été nulle part signalé en Afrique. Cette innovation est peutêtre liée au fait que dans une société multi-raciale anthropologiquement assez mélangée, comme les Etats-Unis, les cheveux crépus sont considérés comme des marqueurs plus forts que la couleur de la peau aux nuances graduées et à la signification peu discrète. Il aurait été mal venu d'inṿoquer différents objets de civilisation, en tant qu'objets du choix primitif fatal, dans une civilisation racialement mixte où l'inégalité sociale entre Noirs et Blancs s'exprime dans des registres infiniment plus subtils, si l'on ose dire, que par la possession d'un livre ou d'un fusil. L'insistance sur le "mauvais choix" des cheveux crépus recouvre en fait une représentation doublement négative et, là encore, quasi-fataliste, du destin des Noirs. Premièrement, les Noirs sont des laissés-pour-compte de la société en tant que groupe racial (crépus) et, deuxièmement, ils sont moins beaux que les Blancs. Ce destin négatif comporte donc non seulement la frustation socioiconomique mais encore une blessure narcissique. I $\mathrm{ce}$ fait que ce destin 
GÖRÖK-KARADY, Veronika. Mythes fondateurs: li. gine de l'inegalite (afrique noire, amerique du nord).

fait suite au mauvais choix à connotation morale plus ou moins forte - les ancêtres pèchent par défaut de modération, de retenue ou de réflexion - et que leur condamnation vient de Dieu, instaurateur suprême de l'ordre du monde aggravent encore le caractère culpabilisant de ces récits. On comprend que les mouvements noirs pour l'égalité des droits civiques aient vigoureusement réagi contre ces représentations idéologiques traditionnelles en retournant l'image négative qu'elles véhiculent, comme en témoigne, par exemple, le slogan "Black is beautiful". Il s'agit là pour eux précisément de rejeter le canon de beauté repris jadis sur la société dominante - celle des Blancs - et d'affirmer le caractère esthétiquement positif de la "négritude" (que symbolise bien la coiffure "afro") ainsi que de revendiquer des chances socio-économiques semblables à celles dont disposent les partenaires blancs.

On raconte aussi en Amérique des récits avec "épreuve de comportement" où les protagonistes ancestraux se rendent coupables d'une faute de conduite. Là encore la source première de ces récits est la Genèse biblique comme c'est le cas en Europe et en Afrique, tel qu'on l'a déjà vu par l'exemple de l'historie des enfants d'Eve. En Amérique on évoque ainsi souvent l'histoire des anges, messagers de Dieu, dont les Noirs ne suivent pas ou suivent avec retard l'appel, ce qui leur vaut leur rejet par Dieu. Attachons-nous à l'examen un peu plus attentif des versions disponibles de l'histoire syncrétique des fils de Noé.

Au sujet des conditions de production des versions africaines de l'histoire des fils de Noé, notamment de celles qui ont été répandues au Tanganyika, nous avons de précieux témoignages, grâce à T. O. Beidelman (Beidelman, 1963). II en ressort que l'interprétation africaine de cette histoire doit beaucoup à l'exégèse européenne qui en a tiré une véritable apologic de la colonisation. Ce parti-pris a fortement marqué l'action des missionnaires de la Church Missionary Society qui continuaient à en faire état dans leurs sermons jusqu'aux années 1960. D'après cette version quasi officielle, la race noire descend de Cham, fils de Noé, coupable de s'être 
Revista da ANPOLL, $\mathrm{n}^{\circ}$ 4, p. 273-298, jan./jun. 1998

moqué de la nudité de son père et frappé à ce titre de la malédiction divine. On peut comprendre de deux manières l'adoption de cette interprétation en milieu africain. II y a d'abord le consensus, largement attesté dans l'ensemble du corpus africain des textes idéologiques, concernant l'infériorité du destin échu aux Noirs et l'attribution de ses causes au méfait de l'ancêtre mythique: c'est le schéma général du bouc émissaire primitif dont on connaît maintes réalisations. On peut ensuite interpréter la relative popularité de cette interprétation de la Bible par le fait qu'elle s'inscrit dans un mouvement de conversion et qu'elle permet aux Chrétiens néophytes de se placer à une distance symbolique décisive par rapport à leurs frères de race non convertis. Dans cette optique la malédiction perd de son efficace pour ceux qui se rangent du côté des élus de Dieu - puisque le sens du christianisme est ici avant tout une association avec la civilisation européenne, celle des Blancs (qui "apportent" le christianisme aux Noirs) - pour ne continuer à frapper que les autres, attachés à leurs croyances anciennes.

Voici la version américaine de la même histoire, dont le texte, donné ici dans une traduction littérale, est émaillé des remarques du narrateur:

\footnotetext{
"Dieu donna la religion à Adam, la reprit de lui pour la donner à Noé. Et vous savez, mademoiselle, Noé avait trois lils et lorsqu'il s'enivra de vin un de ses fils. se moqua de lui; les autres fils ont pris un drap, se sont rapprochés de lui à reculons pour le couvrir: Noé dit à celui qui s'était moqué de lui: Tes enfants seront des bûcherons, ils puiseront de l'eau pour les deux autres fils et ils seront reconnaissables par la chevelure et par la peau foncéc... Ainsi, Mademoiselle, nous voilà maintenant. Dieu a voulu que nous soyons ainsi. Nous avons toujours été obligés d'imiter les Blanes et faire ce que nous les voyons faire. Et voilà tout ce qu'on peut dire. Vous ne pouvez pas changer les paroles de Dieu".
}

On voit que ce récit américain est encore plus explicite dans son message idéologique que le texte africain brodant sur le même thème de l'Ancien Testament. Sur le sens et l'origine de cette adaptation, Patricia Turner propose deux interprétations. Premièrement, il pourrait s'agir du produit de la manipulation de l'enseignement biblique commise par les 
CÖRÖK-KMRADY, Veronika. Mythes fondateurs: lion gine de l'inegalite (afrique noire, amerique du nord).

Blancs du Sud qui profitaient de l'analphabétisme de leurs esclaves pour accréditer cette version de l'histoire portant légitimation de l'ordre esclavagiste établi. pareilles versions auraient été diffusées par les Ėglises également et ceci d'autant plus facilement que les Noirs ne pouvaient connaître l'intégrité du Livre Sacré. Patricia Turner n'exclut pas l'hypothèse que ce fut de propos délibéré que les Noirs du Sud furent privés d'enseignement élémentaire "in order to prevent the slaves from reading the portions of the Bible that stress equality". I Le texte souligne particulièrement l'alićnation des Noirs qui sont non seulement rejetés dans un statut social d'assujettis mais encore condamnés à une sorte de mimétisme des usages des Blancs, ce qui est une manière de leur dénier toute identité sociale authentique. On relèvera ici aussi l'acceptation fataliste de cet état des choses, proclamé avec insistance comme destin immuable.

En contraste avec la majorité des récits à tonalité profondément pessimiste, où l'ancêtre noir figure la négativité, il convient de rappeler l'existence du texte $n^{\circ} 7$ où le Noir fait figure de créature préférée de Dieu et où la couleur noire est présentée comme la plus belle de toutes. C'est dans la même perspective qu'il faut considérer aussi la passage inspiré par la Bible sur le meurtre d'Abel par son frère Caïn ( $n^{\prime \prime 25}$ ). Dans la version afro-américaine de cette histoire c'est en effet le Blanc qui accomplit le crime. Sa transformation en Blanc est précisément due à l'effroi qu'il éprouve devant la menace de punition brandic par Dieu. La race blanche est donc issue d'un criminel, "mauvais gardien de son frère", l'ancêtre noir: Si dans ce récit c'est l'ancêtre blanc qui est accablé du "méfait primitif", on remarquera que le texte ne souffle mot de l'inégalité de conditions sociales qui s'est instaurée entre les groupes raciaux, puisque, logiquement la faute du passé ne saurait justifier une supériorité. L ne narrateur préfère donc passer celle-si sous silence. En somme il n'exploite pas, comme il pourrait le faire, le mauvais rôle attribué au Blanc dans un sens idéologique, par exemple contestataire. Quelles que soient donc les implications de la 
narrative qu'il utilise, le discours afro-américain sur l'inégalité se limite en somme à un discours systématiquement auto-culpabilisant.

De fait, il scrait difficile de tirer autre chose que des traits négatifs de "l'image de soi" qui se dégage de ces récits. Les textes s'accordent à dépeindre les Noirs, représentés par l'ancêtre, sous le signe de la négativité, exclusivement marqués par des défauts.

II s'agit de défauts autant éthiques (manque de respect à Dieu, désobéissance, imprévoyance, insouciance, paresse, manque de diligence, gourmandise, inconstance, etc...) qu'esthétiques (moindre beauté, saleté). On peut distinguer trois aspects de ces images stéréotypées. Tout d'abord on y retrouve aisément les principaux éléments constitutifs des deux structures de conduite opposée, d'une part la rationnalité moderne toute orientée vers des fins de jouissance différée comportant une période consacrée à l'investissement, et d'autre part, la conduite au jour le jour, avec de préférence, l'agrément immédiat et passager - même médiocre - et le refus de tout effort à plus long terme. C'est l'aspect le plus proprement sociologique dans l'auto-perception que véhiculent les récits. II y a aussi, en second lieu, un élément moral qui réside dans la désobéissance (à Dieu, au Père ancestral) exprimée souvent par un défaut de discipline. Or cet élément ne prend tout son sens, que dans une optique patriarcale (qui est aussi l'optique chrétienne) avec une autorité centrale indiscutéc et indiscutable (Dieu, le Père). Toutes ces histoires sont en effet bâties sur une conception absolutiste et ce défi lancé à l'autorité est à son tour une faute absolue. C'est comme cela que s'éclaire la disproportion apparente entre le "crime originel" et son châtiment. Enfin, le troisième aspect négatif de l'image des Noirs est d'ordre esthétique. I La présentation de soi dans ces récits recèle régulièrement une blessure narcissique profonde. C'est là peutêtre le volet le plus étonnant de ces stéréotypes qui n'ont pas de parallèles du moins de façon aussi systématique dans les groupes dominés (chez les Juifs en Europe par exemple, voire chez les Indiens d'Amérique). I Le défaut esthétique reprend en effet le stéréotype européen ethnocentrique et laisse 
GÖRÖK-KARAI)Y, Veronika. Mythes fondateurs: lis igine de linegalite (afrique noire, amerique du nord).

sans compensation symbolique l'assujettissement subi et les autres faiblesses - que ce soit en termes de technicité ou en termes socio-économiques dont les Noirs apparaissent comme accablés dans cette tradition idéologique. C'est là que se confirme avec la plus grande brutalité une image de soi de "damnés de la Tère".

RESUMO: Toda formação social elabora um certo discurso mais ou menos consensual sobre a experiência das desigualdades a fim de mostrar; juslificar ou contestar suas causas. Os discursos sobre a desigualdade nas sociedades sem Iradição escrila objeliva-se na tradição oral. Este taxto apresenta, inicialmente, a história dos filhos de Eva - narrativa difundida na Europa e também conhecida no Brasil e na Âfrica Negra. A seguir são analisados alguns exemplos tirados das civilizaçōes da África Negra e dos Negros da América.

PALAVRAS-CIIAVE: tradição oral; desigualdade; milos fundadores; África negra; América do Norte.

\section{BIBLIOGRAPHIE}

AMRNE, Anti \& Thompson, Stith. The Types of the Folklale. Ilelsink, 1973.

BEIDELMAN, T.O. "A Kaguru Version of the Sons of Noah: a Study in the Inculcation of the Irea of Racial Superiority", Cahiers d'Eludes Africaines 3(4), 1963: 301-326.

DUNDES, Nn. "Slurs International: Folk Comparisons of Ethnicity and National Character", Southem Folklore Quarlely. 39, 1975: 15-38.

CÖRÖG-KARADY, Veronika. Noirs el Blancs. Leur relation dans la littéralure orale africaine. Etude-Anthologie. Paris, SELAF, 1976.

"Reteling Cenesis. The Children of Eve and the Origin of Inequality". pp. 31-44 in V. CörögKarady ed. Genres, Forms, Meanings Essays in Africain Oral Literature. Oxford, IASO, 1982.

TURNER, Patricia. An dey been black ever since. An Examination of Aeliological Narratives in Black Folk Tradition. Berkeley, 1982 (Mémoire inédit). 